\title{
Are Vowels and Consonants Processed Differently? Event-related Potential Evidence with a Delayed Letter Paradigm
}

\author{
Manuel Carreiras' ${ }^{1}$, Margaret Gillon-Dowens ${ }^{1}$, Marta Vergara ${ }^{1}$, \\ and Manuel Perea ${ }^{2}$
}

\begin{abstract}
To investigate the neural bases of consonant and vowel processing, event-related potentials (ERPs) were recorded while participants read words and pseudowords in a lexical decision task. The stimuli were displayed in three different conditions: (i) simultaneous presentation of all letters (baseline condition); (ii) presentation of all letters, except that two internal consonants were delayed for $50 \mathrm{msec}$ (consonants-delayed condition); and (iii) presentation of all letters, except that two internal vowels were delayed for $50 \mathrm{msec}$ (vowels-delayed condition). The behavioral results showed that, for words, re-
\end{abstract}

\section{INTRODUCTION}

In alphabetic languages, consonants and vowels are the pieces of which words are made. Recent data collected from patients and adult readers show that a number of experimental phenomena are modulated by the consonant/vowel status of letters, including behavioral measures (i.e., response times and error percentage), electrophysiological correlates, and the BOLD signal (e.g., see Carreiras, Vergara, \& Perea, 2007; Monaghan \& Shillcock, 2007; Buchwald \& Rapp, 2006; Berent \& Marom, 2005; Peña, Bonatti, Nespor, \& Mehler, 2002; Lee, Rayner, \& Pollatsek, 2001; Caramazza, Chialant, Capasso, \& Miceli, 2000; Cutler, Sebastián-Gallés, SolerVilageliu, \& van Ooijen, 2000 for recent evidence). Taken together, these studies strongly suggest that consonants and vowels may contribute differently to lexical access. The present study further analyzes this issue by examining in detail the time course of consonant/vowel processing_via event-related potentials (ERPs) - in a letter-delay paradigm.

The neuropsychological evidence supporting the distinction between consonants and vowels was initially based on two aphasic patients who exhibited contrasting patterns of errors when producing vowels and consonants (Caramazza et al., 2000): One patient made three

${ }^{1}$ Universidad de La Laguna, Tenerife, Spain, ${ }^{2}$ Universitat de València, Valencia, Spain sponse times in the consonants-delayed condition were longer than in the vowels-delayed condition, which, in turn, were longer than in the baseline condition. The ERPs showed that, starting as early as $150 \mathrm{msec}$, words in the consonants-delayed condition produced a larger negativity than words in vowelsdelayed condition. In addition, there were peak latency differences and amplitude differences in the P150, N250, P325, and N400 components between the baseline and the two letterdelayed conditions. We examine the implications of these findings for models of visual-word recognition and reading. times as many errors in vowels as in consonants, whereas the other patient made five times as many errors in consonants as in vowels. Caramazza et al. (2000) concluded that: (i) vowels and consonants must be categorically distinct at some level of representation, and (ii) different neural mechanisms should be responsible for the processing of vowels and consonants. This double dissociation has also been found in two other patients: Cotelli, Abutalebi, Zorzi, and Cappa (2003) reported a dysgraphic patient with a selective impairment to vowels (13.5\% of errors in vowels, $2 \%$ in consonants), whereas Miceli, Capasso, Benvegnu, and Caramazza (2004) reported an individual with a selective impairment to consonants (98.8\% of incorrectly spelled letters were consonants) (see also Tainturier \& Rapp, 2004; Cubelli, 1991 for additional neuropsychological evidence).

Furthermore, in the literature on normal adults, consonants seem to play a more distinctive role in lexical access, with vowel information constraining lexical selection less tightly than consonant information (Cutler et al., 2000). More specifically, Cutler et al. (2000) showed that, when presented with spoken stimuli and allowed to change one phoneme to make a word from a pseudoword, participants more often alter a vowel than a consonant. Thus, when presented with a pseudoword such as zobra, listeners tend to come up with the word zebra, rather than with the word cobra, indicating that a vowel substitution is easier than a consonant substitution. This phenomenon is independent of the phonemic 
repertoire of a language (Cutler et al., 2000). For example, it occurs in speakers of Spanish, a language which has very few vowels, and thus, an unbalanced consonantto-vowel ratio, and in speakers of Dutch, where the consonant-to-vowel ratio is more balanced because of the large number of vowels. Thus, the results of Cutler et al.'s experiments seem to indicate that the more distinctive role of consonants is independent of the phonemic repertoire of a given language. Indeed, words in Semitic languages are usually written as a series of consonants, with the vowels omitted. Furthermore, information about consonants, but not about vowels, appears to have a crucial role in the detection of words in a continuous speech stream (Bonatti, Peña, Nespor, \& Mehler, 2004; see also Nespor, Peña, \& Mehler, 2003; Peña et al., 2002): Participants appear unable to use transitional probabilities between successive vowels to find words in an artificial stream of continuous speech, even though they have no difficulty doing so using transitional probabilities between successive consonants. In these experiments, the participants were presented with artificial speech streams embodying nonsense words and with rules implemented either over consonants or vowels, and later they were tested on their knowledge of the words of that "language" with a two-alternative forcedchoice paradigm. Participants identified words by assessing statistical dependencies between consonants, but failed to do so when vowels carried the same dependencies.

Recent research with other paradigms suggests that, during visual-word recognition, the processing of consonants is more rapid than that of vowels, providing further evidence for the distinction between consonant and vowel processing (e.g., Lee, Rayner, \& Pollatsek, 2002; Lee et al., 2001; Berent \& Perfetti, 1995). In an influential paper, Berent and Perfetti (1995) found that a target word (rake) was better identified when followed by a consonant-preserving nonword mask (RIKK) than when followed by a vowel-preserving nonword mask $(R A I B)$ at very short exposure durations of targets and masks. Berent and Perfetti interpreted their findings in the framework of a two-cycle model, according to which there is a representational and computational distinction between consonants and vowels. Consonants are computed in the first cycle via a rapid, automatic process, whereas vowels are computed in the second cycle via a slower, controlled process. More recently, Lee et al. (2001, 2002) assessed the early encoding of consonant and vowel information using two paradigms: delayed letter and fast priming. In the delayed-letter paradigm, some of the letters of a target word are initially replaced by dashes at the beginning of an eye fixation during reading, and after a brief period of time (e.g., $30 \mathrm{msec}$ ) the target word appears written normally. Lee et al. (2001) found that the gaze duration on the target word was longer when consonant information was delayed for $30 \mathrm{msec}$ (readers saw $b u-b$ during the first $30 \mathrm{msec}$ of the fixation on bulb) than when vowel information was delayed for $30 \mathrm{msec}$ (readers saw $b$-lb for the first $30 \mathrm{msec}$ of the fixation). In the fast priming paradigm, a string of random letters is initially displayed in the target position (see Sereno \& Rayner, 1992). When the reader's eyes cross an invisible boundary, which is usually located just before the last letter of the word preceding the target word, the random letters are replaced by a prime which remains for a brief period (around $30 \mathrm{msec}$ ) at the beginning of the fixation on the target word. After that period, the prime is replaced by the target word. Lee et al. (2002) found that gaze durations on target words were shorter when preceded by consonant-same word primes (which shared consonant information with the target word; e.g., lake-like) than when preceded by vowel-same word primes (which shared vowel information with the target word; e.g., line-like). Thus, the greater effect for consonants during the initial $30 \mathrm{msec}$ in the Lee et al. experiments strongly suggests that consonants are initially processed more rapidly than vowels.

It is important to note that, despite all the above evidence, none of the current computational models of visual-word recognition (e.g., Gomez, Ratcliff, \& Perea, in press; Dehaene, Cohen, Sigman, \& Vinckier, 2005; Grainger \& van Heuven, 2003; Coltheart, Rastle, Perry, Ziegler, \& Langdon, 2001; Whitney, 2001; Davis, 1999; Grainger \& Jacobs, 1996; McClelland \& Rumelhart, 1981) can account for a differential processing of consonants and vowels. Thus, it is critical to examine in detail the time course of the processing of vowels and consonants during lexical access. Specifically, the main goal of the present experiment was to examine the role of consonants and vowels in lexical access during visual-word recognition by using a letter-delay paradigm combined with the recording of electrophysiological measures. ERPs are functionally decomposable to a greater extent than behavioral data, thus enabling us to draw conclusions not only about the existence of processing differences between vowels and consonants but more importantly, about the level of processing at which these differences occur. For instance, if consonant/vowel differences appear at a very early window (e.g., P150), this would imply that the front-end (i.e., the input coding scheme) of current models of visual-word recognition would need to be modified to assume that the consonant/vowel label occurs at a very early stage. An experiment measuring ERPs is, therefore, particularly helpful in this respect. In particular, we ask: (i) whether consonants and vowels have a differential influence at the early stages of the process of visual word recognition, (ii) what is the time course for the processing of consonants and vowels, and (iii) whether there is an early ERP component sensitive to vowel/consonant status. To this end, we employed a letter-delay paradigm similar to the one used by Lee et al. (2001). In the present experiment, each target item (e.g., CHOCOLATE) was presented in three different conditions: baseline, consonants-delayed, and vowels- 
delayed. In the baseline condition, the word was presented with all its letters from the very beginning (i.e., there was no display change); in the consonants-delayed condition, two nonadjacent internal consonants (e.g., CHO O ATE) were delayed for $50 \mathrm{msec}$ and then the whole word appeared; and finally, in the vowels-delayed condition, two nonadjacent vowels (CHOC L TE) were delayed for $50 \mathrm{msec}$. The complete words were presented in the same position as the words with missing letters.

ERPs are voltage changes recorded from the scalp and extracted from the background electroencephalogram by averaging time-locked responses to stimuli onset. Following Holcomb and Grainger (2006), of specific interest for our study are four components: P150, N250, P325, and N400. Using a masked repetition-priming paradigm, they recorded ERPs to critical items which were repetitions, partial repetitions, or unrelated to the immediately preceding masked prime word. The critical items were nonanimals that were presented together with animal names to which participants were asked to respond. Holcomb and Grainger found a modulation of P150, N250; P325, and N400 components, which they proposed to be related with the sequential overlapping steps of the processing of printed words. The P150 component has a posterior scalp distribution focused over right occipital scalp sites and is larger (i.e., more positive) to target words that are repetitions of a prior prime word, compared to targets that are unrelated to their corresponding prime (Holcomb \& Grainger, 2006). The N250 component with a central-anterior distribution has been associated with the degree of prime-target orthographic overlap (being larger for hoise-HOUSE pairs than for bouse-HOUSE pairs) and phonological overlap-this suggests that the N250 component is sensitive to processing sublexical representations (Grainger, Kiyonaga, \& Holcomb, 2007; Holcomb \& Grainger, 2006). The P325 component with a right posterior distribution has been reported to be larger for repeated compared to unrelated words, but does not set apart repeated versus unrelated pseudowords (Holcomb \& Grainger, 2006). Thus, these authors suggest that this component could correspond to the lexical selection moment when a single-word representation is settled as a unique interpretation of the input. The N400 component is a negative deflection occurring around 400 msec after a word presentation and has been associated with lexical-semantic processing (see Holcomb, Grainger, \& O'Rourke, 2002; Kutas \& Federmeier, 2000). In particular, the amplitude of this negativity is an inverse function of lexical frequency, of lexicality, and of orthographic neighborhood size. The amplitude of the N400 component is greater for low-frequency words than for high-frequency words and for pseudowords than for words (Neville, Mills, \& Lawson, 1992; see also Barber \& Kutas, 2007; Carreiras, Vergara, \& Barber, 2005).

In sum, if the contribution of consonants and vowels in the early stages of visual word recognition is different, we expect the delay of consonants versus vowels to have a differential impact on the ERP components described above. More specifically, we expect a difference in early perceptual components, reflected in the P150 component, between the no-delay and the letter-delayed conditions, the reason being that there is a perceptual change only in the letter-delayed conditions. If the P150 component is sensitive to the degree of perceptual overlap, then the identity (no-delay) condition should produce a greater positivity: There is a greater degree of overlap from the very beginning as compared to the letter-delayed conditions. More important for the present purposes-and taking into account the behavioral findings described above-differences between the consonants-delayed and the vowels-delayed conditions are expected in very early components-this would presumably be reflected in the N250 component (i.e., a component which has been posited to be sensitive to orthographic processing). Finally, early and late lexical differences, if any, between the delay of consonants and vowels, as well as differences between the baseline and the two-letter delay conditions, should be noticeable in the P325 and/or the N400 components.

\section{METHODS}

\section{Participants}

Thirty-one (23 women) undergraduate students from the University of La Laguna participated in the experiment in exchange for course credit. All of them were native Spanish speakers, with no history of neurological or psychiatric impairment, and with normal or correctedto-normal vision. Ages ranged from 18 to 31 years (mean $=$ 23 years). All participants were right-handed, as assessed with an abridged Spanish version of the Edinburgh Handedness Inventory (Oldfield, 1971).

\section{Materials}

We selected 120 Spanish words of 7 to 11 letters (mean length: 9.0 letters) with a mean frequency of 26 per million in the LEXESP Spanish database (range: 4-147; Sebastián-Gallés, Martí, Carreiras, \& Cuetos, 2000). For each word, we created three experimental conditions: (i) the word was preceded by itself for 50 msec (identity condition; e.g., CHOCOLATE-CHOCOLATE); (ii) the word was preceded by itself except that two nonadjacent internal consonants (separated by a vowel) were missing (consonants-delayed condition; $\mathrm{CHO} O \mathrm{O}$ ATE-CHOCO$L A T E$ ); and (iii) the word was preceded by itself except that two nonadjacent internal vowels (separated by a consonant) were missing (vowels-delayed condition; CHOC L TE-CHOCOLATE). The position of the delayed letters was around the word center for both the voweldelayed condition and the consonant-delayed condition (i.e., around Position 5 in the two conditions). Across words, the position of the delayed letters was equated 
across the vowel-delayed and consonant-delayed conditions. It is important to mention that, for all words, the target word was the only legal word that could be generated by filling in the missing letter. For instance, from the sequence $C H O$ O ATE or from the sequence CHOC $L T E$, no word other than CHOCOLATE could be generated by filling in the spaces-this was the case for both the vowel-delayed and the consonant-delayed conditions. These words were extracted from low-density orthographic neighborhoods $(n=0.7$ in the Spanish database; Davis \& Perea, 2005). Coltheart's $N$ (i.e., the number of "orthographic" neighbors; see Coltheart, Davelaar, Jonasson, \& Besner, 1977) refers to the number of words that exist in the language by replacing one letter from an existing word in a given position (e.g., bar, can, and cap are orthographic neighbors of car).

For the purposes of the lexical decision task, we included a set of 120 orthographically legal pseudowords of 7 to 11 letters (mean length $=9.0$ letters). These nonwords had been created by replacing the three initial letters from the experimental words (e.g., the nonword BRACOLATE was created from the word CHOCOLATE), so that a word was not possible even with the missing letters. The manipulation for the nonword stimuli was the same as that for the word stimuli (i.e., identity condition, vowel-delayed condition, and consonant-delayed condition). Three lists of materials were created so that participants saw each target word (or nonword) in only one of the three conditions, and the assignment of the stimuli to conditions was counterbalanced across participants.

\section{Procedure}

Participants were seated comfortably in a darkened sound-attenuated chamber. All stimuli were presented on a high-resolution monitor that was positioned at eye level 80 to $90 \mathrm{~cm}$ in front of the participant. The words were displayed in white lowercase Arial 24 against a dark gray background. Participants performed a lexical decision task: They were instructed to press one of two buttons on the response pad to indicate whether the letter string was a legitimate Spanish word or not. A response button was positioned beneath each thumb. For half of the participants, the right button was used to signal the "Yes" response and left button was assigned the "No" response. For the remaining participants, the order was reversed. The sequence of events in each trial is described as follows. First, a fixation point ("+ ") appeared in the center of the screen for $500 \mathrm{msec}$. This fixation point was followed by a word or a pseudoword that remained on the screen for $450 \mathrm{msec}$. In one third of the trials, all letters appeared on the screen from the very beginning (identity-baseline condition). However, in another third of the trials, two nonadjacent internal consonants were delayed for $50 \mathrm{msec}$ (CHO O ATECHOCOLATE), whereas in the other third of the trials, two nonadjacent internal vowels were delayed (e.g.,
CHOC L TE-CHOCOLATE). Overall, all stimuli were presented for $450 \mathrm{msec}$ - including the 50-msec delay. The trial ended with the participant's response, or $2000 \mathrm{msec}$ after the presentation of the word if the participant had failed to respond. The intertrial interval varied randomly between 1000 and $1300 \mathrm{msec}$. The stimuli were presented in different random order for each participant. Twenty-two warm-up trials, which were not further analyzed, were provided at the beginning of the session and were repeated if necessary. Participants were also asked to avoid eye movements and blinks during the interval starting from the fixation point until response was given. Participants were instructed to favor accuracy over speed in their responses.

\section{EEG Recording and Analyses}

Scalp voltages were collected from $58 \mathrm{Ag} / \mathrm{AgCl}$ electrodes which were mounted in an elastic cap (ElectroCap International, Eaton, $\mathrm{OH}, 10-10$ system). Linked earlobes were used as reference (see Figure 1). Eye movements and blinks were monitored with six further electrodes providing bipolar recordings of the horizontal and vertical electrooculogram (EOG). Interelectrode impedances were kept below $10 \mathrm{k} \Omega$. Electroencephalogram (EEG) data were filtered with an analog bandpass filter of $0.01-100 \mathrm{~Hz}$ and a digital $20-\mathrm{Hz}$ low-pass filter was applied before analysis. The signals were sampled continuously throughout the experiment with a sampling rate of $250 \mathrm{~Hz}$.

Epochs of the EEG corresponding to $750 \mathrm{msec}$ after word onset presentation were averaged and analyzed. Baseline correction was performed using the average EEG activity in the $100 \mathrm{msec}$ preceding the onset of the target stimuli as a reference signal value. Following baseline correction, epochs with simultaneous artifacts in at least 10 channels were rejected. In addition, trials that were not responded to correctly were not included in the analysis. This resulted in the exclusion of approximately $13.7 \%$ of the trials $(7.2 \%$ of word trials and $6.6 \%$ of nonword trials). Separate ERPs were formed for each of the experimental conditions, each of the subjects, and each of the electrode sites.

Six regions of interest were computed out of the 58 electrodes, each containing the mean of a group of electrodes. The regions were (see electrode numbers in Figure 1): left anterior (F1, F3, F5, C1A, C3A, C5A), left central (C1, C3, C5, C1P, C3P, TCP1), left posterior (P1, P3, P5, P1P, P3P, CB1), right anterior (F2, F4, F6, C2A, C2A, C2A), right central (C2, C4, C6, C2P, C4P, TCP2), right posterior (P2, P4, P6, P2P, P4P, CB2).

Mean amplitudes and mean peak latencies were obtained for different time windows. For each window, a repeated measures analysis of variance (ANOVA) was performed, including electrode regions (anterior, central, and posterior), hemisphere (left/right), lexicality (words/ pseudowords), and delay (identity/baseline, delay of 
Figure 1. Schematic flat representation of the 58 electrode positions from which EEG activity was recorded (front of head is at top). Approximate International 10-20 system localizations are indicated by labels. The electrodes colored are those grouped and analyzed in the six critical regions.

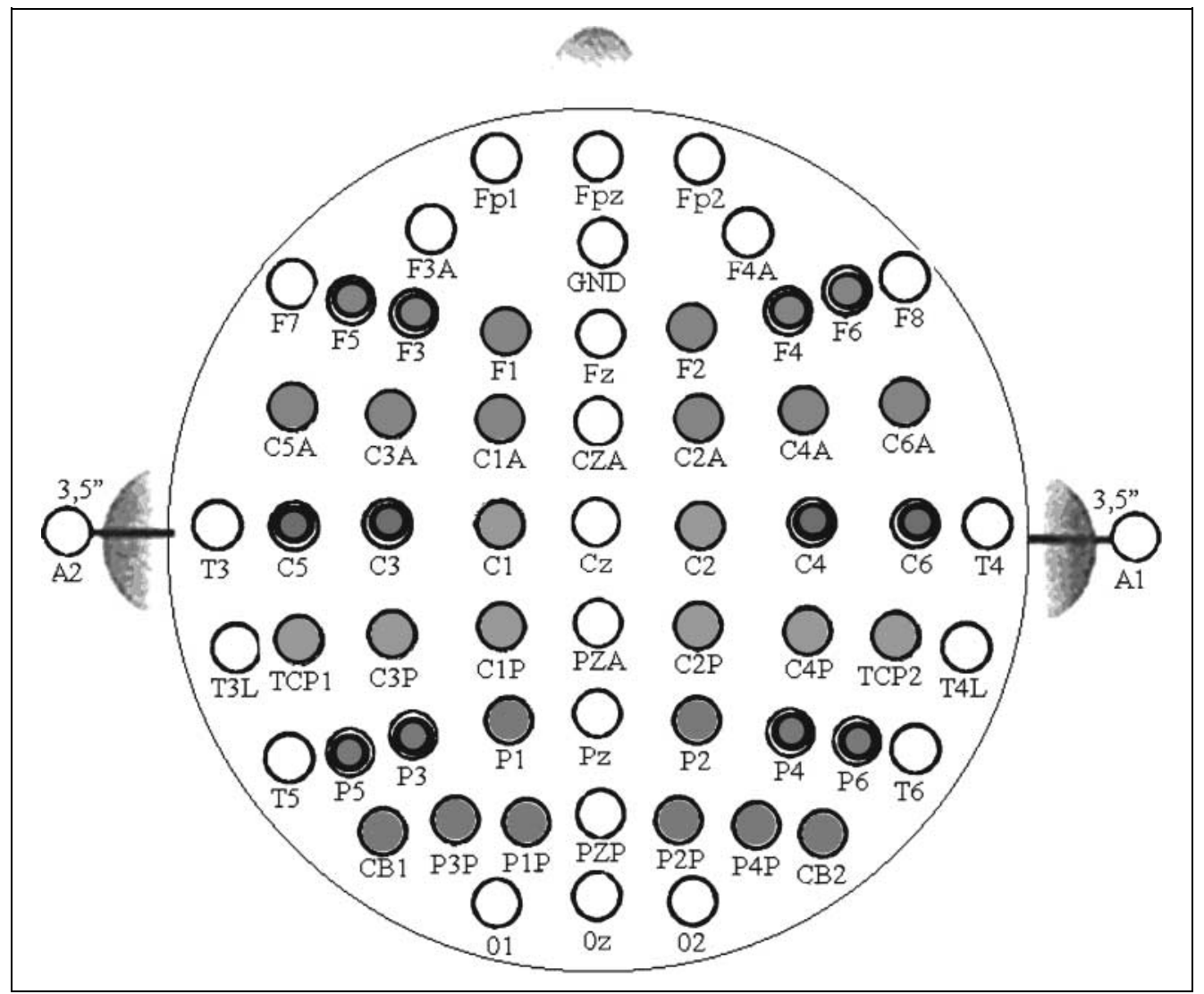

consonants, and delay of vowels) as factors. Where appropriate, critical values were adjusted using the Greenhouse and Geisser (1959) correction for violation of the assumption of sphericity. In addition, post hoc Sidak contrasts were performed after interactions or main effects of delay to control for Type I error in multiple comparisons. Effects for the electrode region factor or for the hemisphere factor will only be reported when they interact with the experimental manipulations.

\section{RESULTS}

\section{Behavioral Measures}

Firstly, reaction times and error rates for word and pseudoword trials were analyzed. Incorrect responses (5.2\% of word trials and 5.1\% of pseudoword trials) were excluded from the latency analysis. In addition, to avoid the influence of outliers, reaction times less than $250 \mathrm{msec}$ or greater than $1500 \mathrm{msec}$ (less than 1.4\% of the data) were also excluded. The mean response times for correct responses and error rates of words and pseudowords are presented in Table 1. Given that the ANOVA on delay (baseline, consonants-delayed, and vowelsdelayed) by lexicality (word, pseudoword) as factors produced a significant interaction in the latency data $[F(2,60)=3.2, p<.05]$, words and pseudowords will be analyzed separately.
For words, the ANOVA showed a significant effect of delay on the response times $[F(2,60)=26.9, p<.001]$. Planned comparisons showed that words with delayed vowels as well as words with delayed consonants were responded to more slowly than words in the baseline condition $[F(1,30)=43.3, p<.001$ and $F(1,30)=44.6$, $p<.001$, respectively]. More importantly, words with delayed consonants were responded to more slowly than words with delayed vowels $[F(1,30)=6.07, p<.02]$. The ANOVA on the percentage of errors did not reveal a significant effect of delay $[F(2,60)=1.1, p=.32]$.

For pseudowords, the ANOVA showed a significant effect of delay on response times $[F(2,60)=3.35$, $p<.05]$. Pseudowords with delayed vowels and pseudowords with delayed consonants were responded to more slowly than pseudowords in the baseline condition $[F(1,30)=4.9, p<.035$ and $F(1,30)=6.1, p<.02$, respectively]. Unlike the word trials, there were no

Table 1. Mean Lexical Decision Times (msec) and Percentage of Errors (in Parentheses) on Words and Pseudowords

\begin{tabular}{llcc}
\hline & Baseline & $\begin{array}{c}\text { Vowels } \\
\text { Delayed }\end{array}$ & $\begin{array}{c}\text { Consonants } \\
\text { Delayed }\end{array}$ \\
\hline Words & $569(5.0)$ & $588(4.8)$ & $601(6.0)$ \\
Pseudowords & $673(5.5)$ & $685(5.1)$ & $688(4.9)$ \\
\hline
\end{tabular}


differences between the consonants-delayed and the vowels-delayed conditions $(F<1)$. The ANOVA on the error rates did not reveal a significant effect of delay $(F<1)$.

\section{Electrophysiological Measures}

ERP grand averages time locked to the onset of the target words and pseudowords are represented in Figures 2 and 3, respectively, over 12 recording sites. Figures 2 and 3 show the three conditions: baseline/ identity, consonants-delayed, and vowels-delayed. Firstly, a negative potential peaking around $100 \mathrm{msec}$ after target completion is observed across the plots. This is followed by a positive potential peaking around $170 \mathrm{msec}$ in posterior regions ( $\mathrm{P} 5, \mathrm{P} 3, \mathrm{P} 4$, and $\mathrm{P} 6)$. Following these peaks, a negativity peaking around $250 \mathrm{msec}$ postonset is observed in central and posterior electrodes, which is followed by a long-lasting positivity peaking around $300 \mathrm{msec}$. These earlier peaks are mainly distributed over posterior areas. The N400 component is observed starting around 350 msec with a center-parietal distribution. Figure 4 shows the typical N400 effect of lexicality: The amplitude of the N400 component is larger for pseudowords than for words. This is so not only for the no-delay (identity) condition (left panel) but also for the two letter-delayed conditions (right panel). In addition, the right panel of Figure 4 also shows a sustained negativity starting at 150 msec, indicating differences between the two letterdelayed conditions in words only.

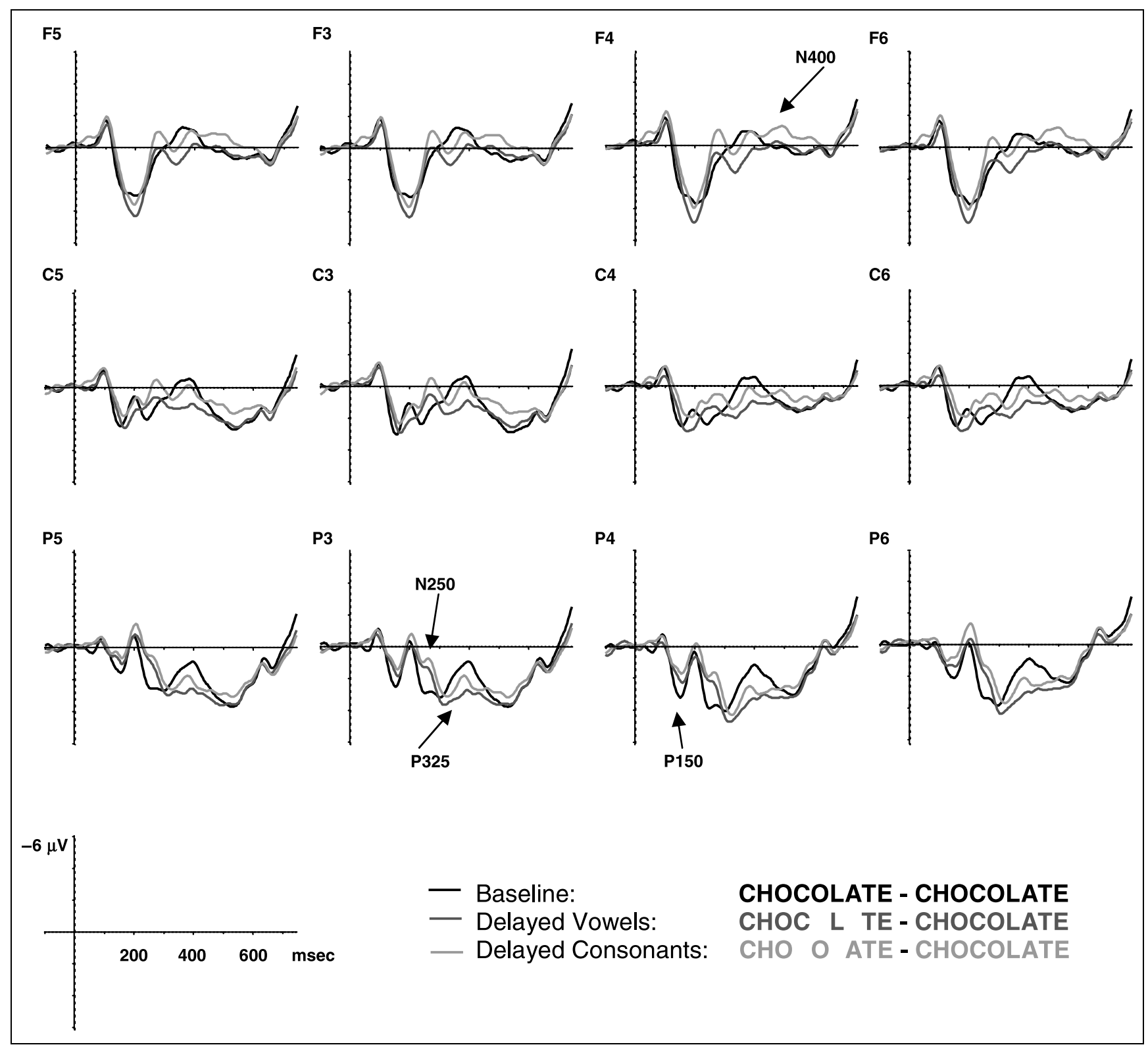

Figure 2. ERP waves for words corresponding to the no-delay, consonants-delayed, and vowels-delayed conditions in 12 representative electrodes. Relative electrode positions are indicated with circles in the schematic head in Figure 1. Negative voltages are plotted up. 


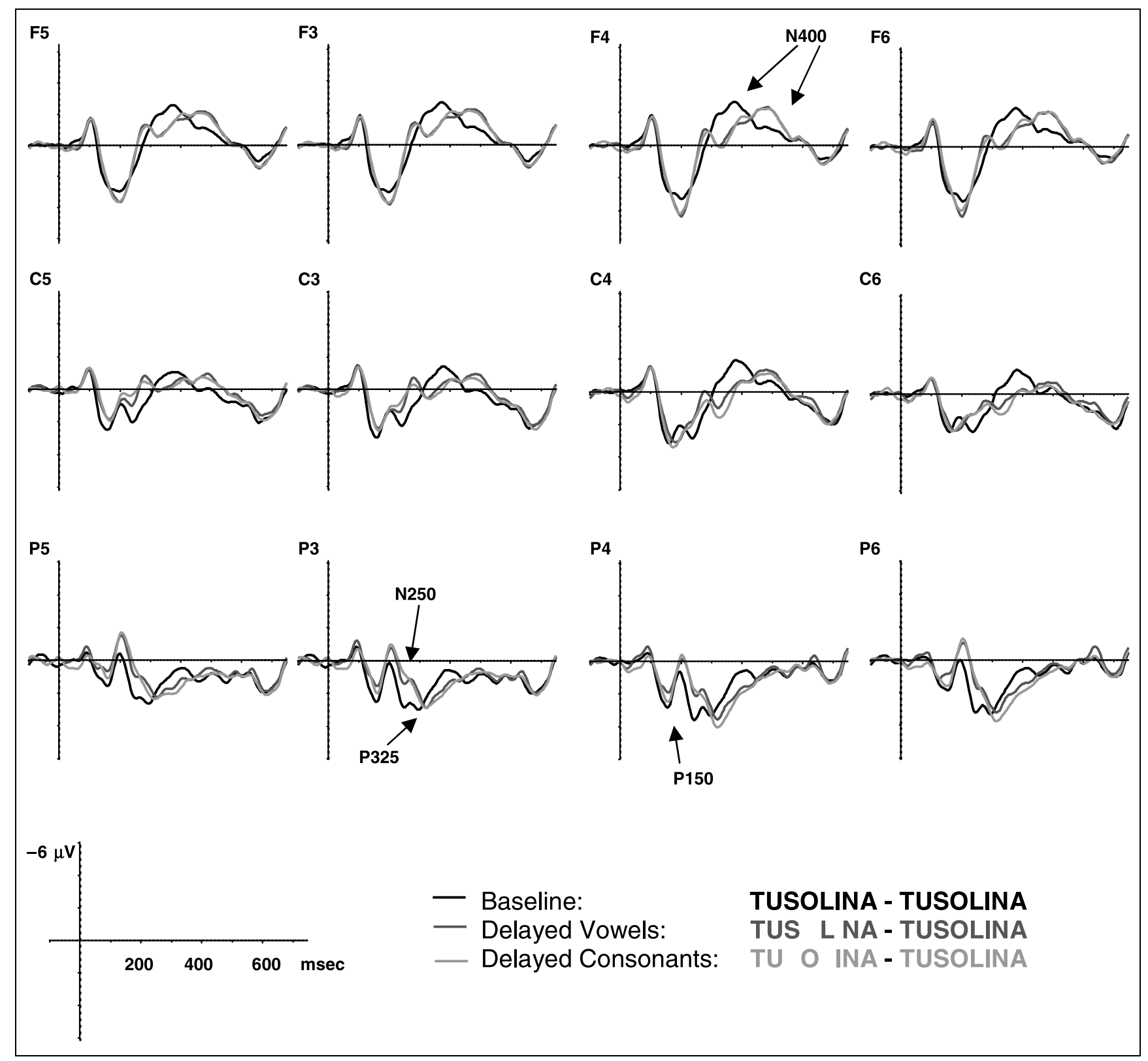

Figure 3. ERP waves for pseudowords corresponding to the no-delay, consonants-delayed, and vowels-delayed conditions in 12 representative electrodes. Relative electrode positions are indicated with circles in the schematic head in Figure 1. Negative voltages are plotted up.

Visual inspection reveals latency and amplitude differences in all windows described. Regarding latency, delayed vowels produce longer latencies than the baseline condition in the P150 and the N250 windows, whereas the two delayed conditions (consonants and vowels) present longer latencies than the baseline condition in the P325 and the N400 windows. Visual inspection of amplitudes shows larger values for the baseline condition than for the letter-delayed conditions in posterior regions in the P150. The next negative peak (N250) shows larger amplitudes for the delayed-letter words compared to the baseline condition. In addition, the consonantdelayed words show larger N250 amplitudes than the vowel-delayed words in more anterior electrodes. Regard- ing the positive component peaking around $300 \mathrm{msec}$ (P325), this shows larger amplitudes for delayed vowels than for the other two conditions for words, and for the two delayed conditions compared to the baseline condition for nonwords. N400 amplitude differences are also observed between words and pseudowords, as well as between consonant delay and baseline. Finally, when specifically comparing the two letter-delayed conditions in the group of words, there is a sustained negativity effect which is larger for the consonants-delayed conditionstarting as early as $150 \mathrm{msec}$ and lasting up to $500 \mathrm{msec}$ (see Figure 4).

Mean amplitude and peak latency values were calculated over four windows of analysis, which were selected 
Figure 4. ERP waves corresponding to the no-delay conditions for words and pseudowords (left) and for the consonants-delayed and vowels-delayed conditions for words and pseudowords (right) in Cz. Negative voltages are plotted up.

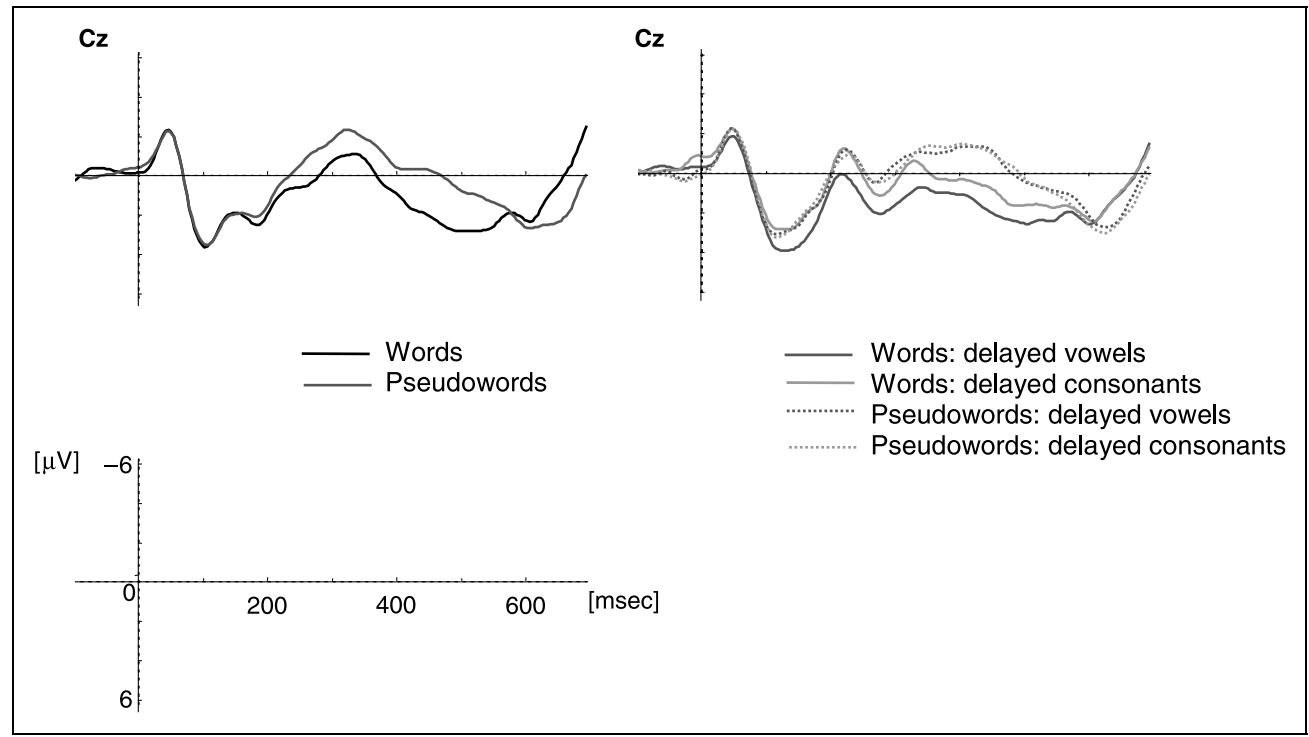

according to visual inspection and taking into account the windows used by Holcomb and Grainger (2006) (P150: 120-170 msec; N250: 200-300 msec; P325: 300400 msec; N400: 400-550 msec). The peaks within each of the windows were calculated as the maximum positive or negative averaged values across each group of six electrodes corresponding to each region of interest (see Figure 5). Mean amplitudes were also calculated for the two delayed conditions, starting from $150 \mathrm{msec}$ until 500 msec. We will describe latency analyses first, followed by amplitude analyses.

\section{Latency Analyses}

P150: 120-170 msec. The ANOVA on the latency values of the P150 peak showed a main effect of delay $[F(2$,

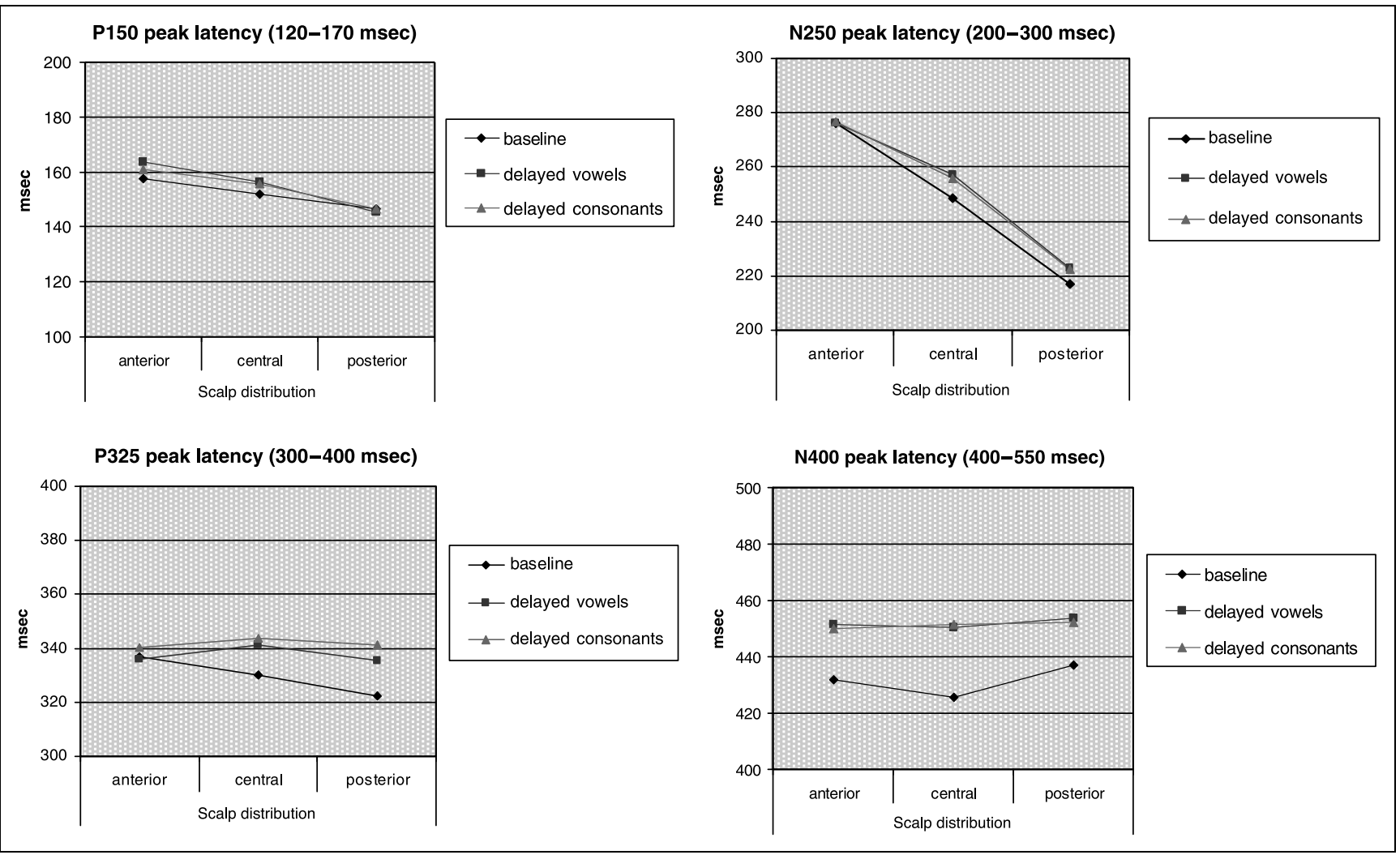

Figure 5. Peak latencies of the P150, N250, P325, and N400 components. 
$60)=3.64, p<.05, \varepsilon=.766]$. The interaction of delay and electrode was significant $[F(4,120)=4.17, p<.01$, $\varepsilon=.792]$. The peak latency of the vowel-delayed condition was larger than that of the baseline/identity condition [vowels-delayed vs. baseline: $F(1,30)=12.6$, $p<.01]$ in anterior electrodes. No other differences between experimental conditions or interactions with lexicality were significant.

N250: 200-300 msec. The ANOVA on the latency values of the N250 peak showed a main effect of delay $[F(2$, $60)=3.4, p=.05, \varepsilon=.721]$. The peak latency of the vowel-delayed condition was larger than that of the baseline/identity condition [vowels-delayed vs. baseline: $F(1,30)=5.22, p<.05]$. There were no differences between the other conditions. None of the interactions with lexicality was significant.

P325: 300-400 msec. The ANOVA on the latency values of the P325 peak showed a main effect of delay $[F(2$, $60)=5.2, p<.05, \varepsilon=.856]$. The interaction of delay and electrode was also significant $[F(4,120)=5.06$, $p<.01, \varepsilon=.718]$. The peak latency of the two letterdelayed conditions was larger than that of the baseline/ identity condition in central and posterior electrodes [central: vowels-delayed vs. baseline: $F(1,30)=10.8$, $p<.01$; consonants-delayed vs. baseline: $F(1,30)=7.1$, $p<.05$; posterior: vowels-delayed vs. baseline: $F(1$, $30)=12.3, p<.01$; consonants-delayed vs. baseline: $F(1,30)=16.2, p<.001]$. None of the other interactions with lexicality was significant.

N400: 400-550 msec. The ANOVA on the latency values of the N400 peak showed a main effect of lexicality $[F(2$, $60)=8.9, p<.01]$ and of delay $[F(2,60)=15.9, p<.01$, $\varepsilon=.841]$. Regarding lexicality, N400 peak latency was larger for pseudowords than for words. Regarding delay, the peak latency of both delayed conditions was larger than the baseline [anterior: vowels-delayed vs. baseline: $F(1,30)=25, p<.001$; consonants-delayed vs. baseline: $F(1,30)=16.6, p<.001]$, whereas no differences were observed between the two delayed conditions [vowelsdelayed vs. consonant-delayed: $F<1$ ].

\section{Amplitude Analyses}

P150: 120-170 msec. The ANOVA on the average values of the 120-170 msec time epoch showed a main effect of delay $[F(2,60)=9.9, p<.001, \varepsilon=.963]$. The amplitude of the P150 component was larger for the baseline than for the two letter-delayed conditions [baseline vs. vowels-delayed: $F(1,30)=12.9, p<.001$; baseline vs. consonants-delayed: $F(1,30)=18.5$, $p<.001]$. No differences were found between the two letter-delayed conditions $(F<1)$. None of the interactions with lexicality was significant.
N250: 200-300 msec. The ANOVA on the average values of the 200-300 msec time epoch showed a main effect of delay $[F(2,60)=15, p<.001, \varepsilon=.801]$. The interaction of delay and electrode was significant $[F(4,120)=6.81$, $p<.01, \varepsilon=.477]$, and so was the interaction of hemisphere, electrode and delay $[F(4,120)=3.3, p<$ $.05, \varepsilon=.891]$. Post hoc comparisons showed that in left anterior, right anterior, and right medial regions, the amplitude of the N250 component was larger for the consonant-delayed than for both the baseline and voweldelayed conditions [left anterior: consonant-delayed vs. baseline: $F(1,30)=17.1, p<.01$; consonant-delayed vs. vowel: $F(1,30)=6.9, p<.03$; right anterior: consonantdelayed vs. baseline: $F(1,30)=24.2, p<.001$; consonantdelayed vs. vowel: $F(1,30)=12.4, p<.01$ and right medial: consonant-delayed vs. baseline: $[F(1,30)=18.4$, $p<.001$; consonant-delayed vs. vowel: $F(1,30)=6.7$, $p<.05]$, whereas in left medial, left posterior, and right posterior areas, the N250 amplitude was larger for the two delayed conditions than for the baseline condition, but no differences were found between the two delayed conditions [left medial: vowel-delayed vs. baseline: $F(1$, $30)=6.6, p<.05$; consonant-delayed vs. baseline: $F(1$, $30)=32.8, p<.001$; left posterior: vowel-delayed vs. baseline: $F(1,30)=13.7, p<.01$; consonant-delayed vs. baseline: $F(1,30)=32.8, p<.001$; right posterior: voweldelayed vs. baseline: $F(1,30)=10.2, p<.01$; consonantdelayed vs. baseline: $F(1,30)=22.0, p<.001]$. None of the interactions with lexicality was significant.

P325: 300-400 msec. The ANOVA on the average values of the 300-400 msec time epoch showed a main effect of lexicality $[F(1,30)=21, p<.001]$ with larger positive amplitudes for words compared to pseudowords; a main effect of delay $[F(2,60)=14.2, p<.005, \varepsilon=.938]$, and an interaction between lexicality and delay $[F(2,60)=$ $3.7, p<.05, \varepsilon=.938$ ]. For words, the amplitude of the P325 component was larger for the vowel-delayed than for both the baseline condition [vowel-delayed vs. baseline: $F(1,30)=19.6, p<.01$ ] and the consonantdelayed condition [vowel-delayed vs. consonant-delayed: $F(1,30)=9.9, p<.05]$ No other significant differences were observed. For pseudowords, the amplitude of the P325 component was larger for both the vowel-delayed and the consonant-delayed condition than for the baseline [vowel-delayed vs. baseline: $F(1,30)=6.41, p<$ .05 ; consonant-delayed vs. baseline: $F(1,30)=12.9$, $p<.05$ ]. No other significant differences were observed.

N400: 400-550 msec. The ANOVA on the average values of the 400-550 msec time epoch showed a main effect of lexicality $[F(1,30)=38.2, p<.001]$ with larger amplitudes for pseudowords compared to words. The interaction of delay and electrode was also significant $[F(4,120)=12.7, p<.001, \varepsilon=.593]$. The amplitude of the N400 component was larger for the consonantdelayed than for the baseline condition [consonant- 
delayed vs. baseline: $F(1,30)=6.7, p<.05$ ] in anterior regions. No other significant differences were observed.

150-500 segment. The ANOVA on the average values of the 150-500 msec time epoch for the two letter-delayed conditions showed a main effect of lexicality $[F(1,30)=$ $22.8, p<.001]$, a main effect of delay $[F(1,30)=4.2$, $p<.05]$, and an interaction between lexicality and delay $[F(1,30)=4.4, p<.05]$. The effect of delay only occurred for words $[F(1,30)=8.9, p<.01$; pseudowords: $F<1$ ]; this effect of delay for word stimuli was due to a larger sustained negativity for consonantdelayed words than for vowel-delayed words $[F(1,30)=$ $8.9, p<.01]$. None of the other interactions with lexicality was significant.

\section{DISCUSSION}

The results of this letter-delay experiment are clear-cut. Firstly, the behavioral data show that delaying two internal letters for $50 \mathrm{msec}$ slows down word identification and, more importantly, that this cost is higher when these letters are consonants (CHO O ATE-CHOCOLATE) - as compared to vowels (CHOC L TE-CHOCOLATE). For pseudowords, we also found an effect of delay; however, unlike the word trials, there were no differences between consonant-delayed and vowel-delayed pseudowords. Secondly, the delay of two letters had an important impact on the peak latency analyses, showing larger latencies for the letter-delayed conditions than for the baseline condition in all windows (P150, N250, P325, and N400)-note that the differences between consonants-delayed and baseline did not reach significance in the two earlier windows (P150 and N250). In addition, there were no differences in peak latency between delay of consonants and vowels. Thirdly, peak latencies for pseudowords were larger than for words in the N400 window. Fourthly, in the amplitude analyses for both words and pseudowords, we found differences between the two letter-delayed conditions and the baseline (identity condition) in the P150 component-that is, the perceptual system seems to detect the presence of a perceptual change in the two letter-delayed conditions. Fifthly, differential effects of amplitude were obtained in the N250 component for the three conditions - that is, there was an early effect of consonant/vowel status. These effects had a different pattern in anterior and posterior regions. In posterior regions, the two letter-delay conditions showed increased amplitude as compared to the baseline, but in anterior regions delaying two consonants resulted in larger amplitudes than delaying two vowels. Sixthly, differences in amplitude were also observed in the P325 window, with a slightly different pattern for words and pseudowords. For pseudowords, the two letter-delay conditions were more positive-going than the baseline. However, for words, the vowels-delayed condition was more positivegoing than the consonants-delayed and the baseline conditions. Seventhly, as expected, amplitude for pseudowords was larger than for words and for the consonantdelay condition as compared to the baseline condition. Finally, a sustained negativity was found only for word stimuli, and this negativity was larger when two consonants were delayed as compared to when two vowels were delayed - this sustained negativity started at $150 \mathrm{msec}$ and lasted up to $500 \mathrm{msec}$. We now examine in detail how the different components of the event related potentials may have been affected by consonant/vowel status during lexical access, and finally, we consider the important implications of the present findings for models of visual-word recognition and reading.

Importantly, the peak latency analyses, showing longer latencies for the two letter-delayed conditions as compared to the baseline/identity condition, suggest that the delay in delivering all the featural information (i.e., two missing letters) of the stimuli to the visualword recognition system appears to cause a delay in the lexical/semantic access to the whole-word form and meaning for word stimuli. This seems to be particularly clear in the two later windows: P325 and N400. In addition, the N400 latencies for pseudowords were larger than the latencies for words. What we should note is that the P325 component has been associated with lexical selection (Holcomb \& Grainger, 2006), whereas the N400 component has been associated with late lexical-semantic processing (e.g., Holcomb et al., 2002; Kutas \& Federmeier, 2000). Our data on the peak latencies are consistent with this idea: The P325 and N400 components may be tapping lexical and lexical/semantic processes. Nonetheless, the latency analyses should be interpreted with some caution when the two delayed conditions differ from the baseline in both words and pseudowords. The reason is that this difference could be also due to an overlap with delayed early visual components. Although the baseline condition involves a single visual presentation, the delayed conditions, by their nature, involve two visual events - the onset of the original, partial stimulus and then the onset of the additional letters. We believe, however, that this argument does not limit the implications of the present experiment because it does not apply when effects were different for words and pseudowords or when the pattern of data differed between the baseline and one type of letter (consonants but not vowels or vice versa), and furthermore, because we found significant differences in direct contrast between consonants and vowels.

In previous studies, the P150 component has been seen to be sensitive to the degree of overlap between primes and targets in a masked priming paradigm (e.g., see Holcomb \& Grainger, 2006). Holcomb and Grainger (2006) found greater positivities for identity than unrelated targets, and they also found greater positivities for targets that completely overlapped their primes in each letter position, and intermediate positivities for targets that overlapped in most, but not all, positions. In the 
present experiment, the P150 component was sensitive to letter delay for both words and pseudowords; however, there were no differences between the consonantdelayed and vowel-delayed stimuli. That is, the P150 component does not seem to be sensitive to consonant/ vowel status. Holcomb and Grainger proposed that the P150 may reflect an early orthographic processing of letters (or forms), which is sensitive to elemental features of stimuli. Consistent with Holcomb and Grainger, our data suggest that the P150 component is sensitive to the temporary delay of letters, independently of whether they are consonants or vowels. Thus, this component may be a correlate of an abstract representation of letters (or of letter features), but if so, it does not appear to be sensitive to the consonant/vowel status of letters. Alternatively, this component could simply reflect an early visual/perceptual effect.

We now turn to the N250 component. The N250 component seems to be sensitive to word processing at the interface between sublexical and whole-word representations (Kiyonaga, Grainger, Midgley, \& Holcomb, 2007). In particular, it may be involved in the processing of the relative position of letters, and combination of letters, such as bigrams and trigrams. For instance, in a masked priming procedure, the $\mathrm{N} 250$ component is more negative to targets that are unrelated to the masked prime word than those that are repeats of the prime, and this component has also shown differential sensitivity, depending on the degree of orthographic overlap between primes and targets (see Kiyonaga et al., 2007; Holcomb \& Grainger, 2006). In the present experiment, the amplitude of the N250 component was larger for the two letter-delayed conditions than for the nondelay (identity) condition in posterior regions. But more importantly, the amplitude of the N250 component was larger when delaying two consonants than when delaying two vowels in anterior regions. And this was so for both words and pseudowords. Thus, the present data strongly suggest that the N250 component is also sensitive to letter identity (via consonant/vowel status) because the effects in the anterior region in this component are different when there is a delay of consonants versus vowels. One possibility is that the delay of two consonants may cause the stimuli to be more distant in terms of perceptual similarity from the whole-word form. (Note, however, that there was only one possible word candidate in each stimulus for the two letter-delayed conditions.) If that is the case, differences in the N250 component in anterior regions could be of similar nature to those reported by Kiyonaga et al. (2007) and Holcomb and Grainger (2006) with partial repetitions (e.g., teble-TABLE) and with pseudoword repetitions, reflecting processing at the sublexical to word processing level.

Another possible interpretation is that the posterior N250 effect is due to purely orthographic-perceptual processes, whereas the more anterior-oriented N250 component is due to late, sublexical effects (e.g., phonological computation). Indeed, Grainger et al. (2007) observed two distinct N250 patterns when manipulating pseudohomophone and transposed-letter primes in a masked priming paradigm. They found that, at the N250 epoch, transposed-letter priming started approximately 50 msec earlier than the pseudohomophone priming and the scalp distribution of these effects was more posterior-oriented for transposed-letter priming and more anterior-oriented for pseudohomophone priming. In the present experiment, the N250 component was larger when delaying consonants as compared to the baseline in anterior regions, but not when delaying vowels as compared to the no-delay condition. That is, the delay of vowels seems to be less detrimental for the visual-word recognition system than the delay of consonants. As indicated in the Introduction, consonant information seems to be more important than vowel information for lexical processing (e.g., Perea \& Carreiras, 2006; Perea \& Lupker, 2004; Lee et al., 2001, 2002; Berent \& Perfetti, 1995) and lexical acquisition (Bonatti et al., 2004; Nespor et al., 2003; Peña et al., 2002). Therefore, the more anterior N250 effects may well be reflecting this sublexical difficulty, whereas the posterior N250 component may also reflect some earlier perceptualorthographic processes. The fact that the delay of vowels differs from the no-delay condition in the posterior, but not in the anterior, regions is consistent with this interpretation.

Another important finding from the present experiment is the amplitude difference in the P325 component. For words, the amplitude was larger when vowels were delayed than in the other two conditions. One possible interpretation of this is that delaying two vowels leaves unpronounceable primes, while this is not the case when delaying two consonants. Delaying consonants will leave a pronounceable string of letters. This fact might inhibit phonological processing when vowels are delayed. Some previous studies have reported differences for phonologically legal and illegal letter strings. For instance, Bentin, Mouchetant-Rostaing, Giard, Echallier, and Pernier (1999) showed that, in the same time range (300-400 msec), unpronounceable consonant letter strings produced a positivity compared to pronounceable pseudowords - which produced a negativity. They suggested that these effects could represent an early lexical or prelexical process of grapheme-to-phoneme translation. However, this interpretation would predict similar effects for words and for pseudowords, which was not the case in the present experiment. The effects for pseudowords in the P325 component were observed between the baseline and the two letter-delayed conditions, but no differences were found between the consonants-delayed and vowels-delayed pseudowords. This dissociation between the word/nonword letter delay conditions seems to imply that not only are sublexical processes influencing the consonant/vowel differences in the P325 component 
but there is also some top-down lexical influence. Indeed, Holcomb and Grainger (2006) suggested that the P325 component may be sensitive to processing within whole-word representations and this component might correspond to when a single whole-word representation is settled as a unique interpretation of the input (i.e., lexical selection). Thus, the observed P325 effects may have been produced by phonological processes, but of a postlexical nature. This would be consistent with the presence of late (postlexical) phonological effects in the N400 time range in a rhyming task (e.g., Praamstra, Meyer, \& Levelt, 1994; Rugg \& Barrett, 1987; Rugg, 1984). Interestingly, in a lexical decision task with auditory stimuli, differences between nonrhyming and rhyming conditions were obtained for words, but not for nonwords (see Praamstra et al., 1994). The presence of phonological effects in a late component for words (but not for nonwords) with the lexical decision task employed by Praamstra et al. (1994) — the task that has been used in the present experiment-suggests that these effects may be postlexical in nature. In any case, more research should be devoted to examining the lexical versus postlexical nature of the phonological processes that may be driving the P325 component.

In the N400 component, we found the traditional pattern, consisting of a larger amplitude for pseudowords than for words (e.g., Carreiras et al., 2005; see Barber \& Kutas, 2007 for a review). The N400 component has been associated with late lexical-semantic processing (e.g., Holcomb et al., 2002; Kutas \& Federmeier, 2000). Therefore, our data are consistent with this idea. In addition, we found larger amplitude for the delay of consonants as compared to the baseline in anterior regions, and this is also consistent with this component being associated with lexical-semantic processing. As indicated in the Introduction, it has been suggested that consonants are more critical for lexical access (e.g., Carreiras et al., 2007; Lee et al., 2001, 2002; Berent \& Perfetti, 1995) and, therefore, the delay of consonants would hinder the process of lexical access.

Finally, a very robust result emerges-only for word stimuli-when contrasting directly the two letter-delayed conditions in a long window lasting between 150 and 500 msec: Delaying two consonants generates a long sustained negativity when compared to delaying two vowels. As stated earlier, it has been hypothesized that vowels and consonants play different roles in lexical processing and in language acquisition. In particular, Nespor et al. (2003) proposed that although the main role of consonants concerns the lexicon, the main role of vowels is to allow the identification of the rhythmic class and the specific properties of syntactic structure. Furthermore, behavioral and eye movement experiments suggest that information about consonants is more critical in accessing the whole-word form (see Carreiras et al., 2007; Perea \& Carreiras, 2006; Perea \& Lupker, 2004; Lee et al., 2001, 2002; Berent \& Perfetti, 1995; but see
Colombo, 2000; Lukatela \& Turvey, 2000). Thus, the sustained negativity for the delay of consonants as compared to the delay of vowels suggests that the delay of consonants is more detrimental for lexical processing. This interpretation is compatible with the idea that the assignment of a consonant label occurs earlier in processing than the assignment of a vowel label (Berent \& Perfetti, 1995). Importantly, the onset of the sustained negativity starts as early as $150 \mathrm{msec}$, and this is consistent with the early differences between consonants and vowels reported in gaze duration (around 300-350 msec) in reading experiments when the participants' eye movements are monitored (Lee et al., 2001, 2002).

We now discuss briefly the issue of the difference in processing between consonants and vowels. Although this difference is assumed to be qualitative by a number of authors (Caramazza et al., 2000; Berent \& Perfetti, 1995), this assumption may not be completely necessary (see Monaghan \& Shillcock, 2003, 2007, for computational evidence; but see Knobel \& Caramazza, 2006). There is an alternative (and apparently simpler) view of the observed consonant/vowel differences. This is in terms of a letter-frequency account: Vowels tend to be more frequent than consonants at the letter identity level. (Note that, in most languages, there are more consonants than vowels, but vowels are more frequent.) Thus, one might argue that the observed differences in the present experiment may be due to letter frequency rather than consonant/vowel status. However, the present data are difficult to explain on the basis of a letterfrequency account (e.g., Lupker, Perea, \& Davis, 2008). This account would predict that vowels, which are more frequent than consonants, should be processed faster. This does not seem to be the case: The delay of consonants-which means that more vowels are available from the beginning-increases reaction times and results in a sustained negativity. If letter frequency (at a letter-identity level) is playing a role here, we should have obtained the reverse effect-note that an increase of negativity is typically associated with the processing of less frequent stimuli. Another finding which would be difficult to explain by a letter-frequency account is that differences in sustained negativity between consonants and vowels were only found when the stimuli were words, but not when they were pseudowords. If letter frequency-rather than differences in consonant/ vowel status-was the key factor, this sustained negativity should have occurred for both word and pseudoword stimuli.

In sum, the present experiment, by using electrophysiological measures, provides clear empirical evidence of a processing distinction between consonants and vowels. Undoubtedly, the consonant/vowel dissociation has important implications for models of visual-word recognition and reading. At present, this processing distinction between vowels and consonants is problematic for all computational and neurophysiological models 
of visual-word recognition (e.g., Gomez et al., in press; Dehaene et al., 2005; Grainger \& van Heuven, 2003; Coltheart et al., 2001; Whitney, 2001; Davis, 1999; Grainger \& Jacobs, 1996; McClelland \& Rumelhart, 1981): None of these models-for the sake of parsimony_distinguish between vowel and consonant processing, and thus, they cannot explain the observed differences between the delay of consonants vs. vowels. Alternatively, the models of Berent and Perfetti (1995) and Caramazza and Miceli (1990), which do consider a differential role of consonants and vowels in lexical access, would need to be implemented computationally to assess their fit to the data. (Bear in mind that computational models have many advantages over "verbal models"; see Monaghan \& Shillcock, 2007; Coltheart et al., 2001.) Future implementations of models of visual-word recognition should take into account that consonants and vowels impose different constraints on the processes of lexical access.

\section{Acknowledgments}

This research was partially supported by grants from the Spanish Ministry of Education and Science (SEJ2004-07680-C02-02/ PSIC, SEJ2005-05205/EDU; SEJ2006-09238).

Reprint requests should be sent to Manuel Carreiras, Departamento de Psicología Cognitiva, Campus de Guajara, Universidad de La Laguna, 38205-Tenerife, Spain, or via e-mail: mcarreir@ull.es; Web: www.neurocog.ull.es.

\section{REFERENCES}

Barber, H., \& Kutas, M. (2007). Interplay between computational models and cognitive electrophysiology in visual word recognition. Brain Research Reviews, 53, 98-123.

Bentin, S., Mouchetant-Rostaing, Y., Giard, M. H., Echallier, J. F., \& Pernier, J. (1999). ERP manifestations of processing printed words at different psycholinguistic levels: Time course and scalp distribution. Journal of Cognitive Neuroscience, 11, 35-60.

Berent, I., \& Marom, M. (2005). The skeletal structure of printed words: Evidence from the Stroop task. Journal of Experimental Psychology: Human Perception and Performance, 31, 328-338.

Berent, I., \& Perfetti, C. A. (1995). A rose is a REEZ: The two cycles model of phonology assembly in reading English. Psychological Review, 102, 146-184.

Bonatti, L. L., Peña, M., Nespor, M., \& Mehler. J. (2004). Linguistic constraints on statistical computations: The role of consonants and vowels in continuous speech processing. Psychological Science, 16, 451-459.

Buchwald, A., \& Rapp, B. (2006). Consonants and vowels in orthographic representation. Cognitive Neuropsychology, 23, 308-337.

Caramazza, A., Chialant, D., Capasso, D., \& Miceli, G. (2000). Separable processing of consonants and vowels. Nature, 403, 428-430.

Caramazza, A., \& Miceli, G. (1990). The structure of graphemic representations. Cognition, 37, 243-297.

Carreiras, M., Vergara, M., \& Barber, H. (2005). Early ERP effects of syllabic processing during visual word recognition. Journal of Cognitive Neuroscience, 17, 1803-1817.
Carreiras, M., Vergara, M., \& Perea, M. (2007). ERP correlates of transposed-letter similarity effects: Are consonants processed differently from vowels? Neuroscience Letters, 419, 219-224.

Colombo, L. (2000). The assembly of phonology in Italian and English: Consonants and vowels. In A. Kennedy, R. Radach, D. Heller, \& J. Pynte (Eds.), Reading as a perceptual process (pp. 377-398). Amsterdam: Elsevier.

Coltheart, M., Davelaar, E., Jonasson, J. T., \& Besner, D. (1977). Access to the internal lexicon. In S. Dornic (Ed.), Attention and performance VI. New York: Academic Press.

Coltheart, M., Rastle, K., Perry, C., Ziegler, J., \& Langdon, R. (2001). DRC: A dual-route cascaded model of visual word recognition and reading aloud. Psychological Review, 108, 204-256.

Cotelli, M., Abutalebi, J., Zorzi, M., \& Cappa, S. F. (2003). Vowels in the buffer: A case study of acquired dysgraphia with selective vowel substitutions. Cognitive Neuropsychology, 20, 99-114.

Cubelli, R. (1991). A selective deficit for writing vowels in acquired dysgraphia. Nature, 353, 258-260.

Cutler, A., Sebastián-Gallés, N., Soler-Vilageliu, O., \& van Ooijen, B. (2000). Constraints of vowels and consonants on lexical selection: Cross-linguistic comparisons. Memory $\mathcal{E}$ Cognition, 28, 746-755.

Davis, C. J. (1999). The Self-Organising Lexical Acquisition and Recognition (SOLAR) model of visual word recognition. Unpublished doctoral dissertation, University of New South Wales.

Davis, C. J., \& Perea, M. (2005). BuscaPalabras: A program for deriving orthographic and phonological neighborhood statistics and other psycholinguistic indices in Spanish. Behavior Research Methods, 37, 665-671.

Dehaene, S., Cohen, L., Sigman, M., \& Vinckier, F. (2005). The neural code for written words: A proposal. Trends in Cognitive Sciences, 9, 335-341.

Gomez, P., Ratcliff, R., \& Perea, M. (in press). The overlap model: A model of letter position coding. Psychological Review.

Grainger, J., \& Jacobs, A. M. (1996). Orthographic processing in visual word recognition: A multiple read-out model. Psychological Review, 103, 518-565.

Grainger, J., Kiyonaga, K., \& Holcomb, P. J. (2007). The time course of orthographic and phonological code activation. Psychological Science, 17, 1021-1026.

Grainger, J., \& van Heuven, W. J. B. (2003). Modeling letter position coding in printed word perception. In P. Bonin (Ed.), The mental lexicon (pp. 1-23). New York: Nova Science.

Greenhouse, S., \& Geisser, S (1959). On methods in the analysis of profile data. Psychometrika, 24, 95-112.

Holcomb, P. J., \& Grainger, J. (2006). On the time course of visual word recognition: An event-related potential investigation using masked repetition priming. Journal of Cognitive Neuroscience, 18, 1631-1643.

Holcomb, P. J., Grainger, J., \& O'Rourke, T. (2002). An electrophysiological study of the effects of orthographic neighborhood size on printed word perception. Journal of Cognitive Neuroscience, 14, 938-950.

Kiyonaga, K., Grainger, J., Midgley, K. J., \& Holcomb, P. J. (2007). Masked cross-modal repetition priming: An event related potential investigation. Language and Cognitive Processes, 22, 337-376.

Knobel, M., \& Caramazza, A. (2006). Evaluating computational models in cognitive neuropsychology: The case from the consonant/vowel distinction. Brain and Language, 100, 95-100. 
Kutas, M., \& Federmeier, K. D. (2000). Electrophysiology reveals semantic memory use in language comprehension. Trends in Cognitive Sciences, 4, 463-470.

Lee, H.-W., Rayner, K., \& Pollatsek, A. (2001). The relative contribution of consonants and vowels to word identification during reading. Journal of Memory and Language, 44, 189-205.

Lee, H.-W., Rayner, K., \& Pollatsek, A. (2002). The processing of consonants and vowels in reading: Evidence from the fast priming paradigm. Psychonomic Bulletin \& Review, 9, 766-772.

Lukatela, G., \& Turvey, M. T. (2000). An evaluation of the two cycles model of phonology assembly. Journal of Memory and Language, 42, 183-207.

Lupker, S. J., Perea, M., \& Davis, C. J. (2008). Transposed letter priming effects: Consonants, vowels and letter frequency. Language and Cognitive Processes, 23, 93-116.

McClelland, J. L., \& Rumelhart, D. E. (1981). An interactive activation model of context effects in letter perception: Part 1. An account of basic findings. Psychological Review, 88, 375-407.

Miceli, G., Capasso, R., Benvegnu, B., \& Caramazza, A. (2004). The categorical distinction of consonant and vowel representations: Evidence from dysgraphia. Neurocase, 10, 109-121.

Monaghan, P., \& Shillcock, R. C. (2003). Connectionist modelling of the separable processing of consonants and vowels. Brain and Language, 86, 83-98.

Monaghan, P., \& Shillcock, R. C. (2007). Levels of description in consonant/vowel processing: Reply to Knobel and Caramazza. Brain and Language, 100, 101-108.

Nespor, M., Peña, M., \& Mehler, J. (2003). On the different roles of vowels and consonants in speech processing and language acquisition. Lingue e Linguaggio, 2, 221-247.

Neville, H. J., Mills, D. L., \& Lawson, D. S. (1992). Fractionating language: Different neural subsystems with different sensitive periods. Cerebral Cortex, 2, 244-258.
Oldfield, R. C. (1971). The assessment and analysis of handedness: The Edinburgh inventory. Neuropsychologia, 9, 97-113.

Peña, M., Bonatti, L. L., Nespor, M., \& Mehler, J. (2002). Signal-driven computations in speech processing. Science, 298, 604-607.

Perea, M., \& Carreiras, M. (2006). Do transposed-letter similarity effects occur at a syllable level? Experimental Psychology, 53, 308-315.

Perea, M., \& Lupker, S. J. (2004). Can CANISO activate CASINO? Transposed-letter similarity effects with nonadjacent letter positions. Journal of Memory and Language, 51, 231-246.

Praamstra, P., Meyer, A. S., \& Levelt, W. J. M. (1994). Neurophysiological manifestations of phonological processing: Latency variations of a negative ERP component time-locked to phonological mismatch. Journal of Cognitive Neuroscience, 6, 204-219.

Rugg, M. D. (1984). Event-related potentials and the phonological processing of words and nonwords. Neuropsychologia, 22, 435-443.

Rugg, M. D., \& Barrett, S. E. (1987). Event-related potentials and the interaction between orthographic and phonological information in a rhyme-judgment task. Brain and Language, 32, 336-361.

Sebastián-Gallés, N., Martí, M. A., Carreiras, M., \& Cuetos, F. (2000). LEXESP: una base de datos informatizada del español. Universitat de Barcelona, Spain.

Sereno, S. C., \& Rayner, K. (1992). Fast priming during eye fixations in reading. Journal of Experimental Psychology: Human Perception and Performance, 18, 173-184.

Tainturier, M. J., \& Rapp, B. C. (2004). Complex graphemes as functional spelling units: Evidence from acquired dysgraphia. Neurocase, 10, 122-131.

Whitney, C. (2001). How the brain encodes the order of letters in a printed word: The SERIOL model and selective literature review. Psychonomic Bulletin E Review, 8, 221-243. 